\title{
EVALUACIÓN DE ZONA DADORA EN RECONSTRUCCIÓN AURICULAR DE MICROTIA CON TÉCNICA SUPRAPERICÓNDRICA EN LA OBTENCIÓN DE CARTÍLAGO COSTAL*
}

\author{
Drs. Silvana Acosta ${ }^{1}$, Carolina Lagos ${ }^{2}$, Ricardo Yáñez ${ }^{1}$
}

1 Sección de Cirugía Plástica y Reconstructiva, División de Cirugía, Pontificia Universidad Católica de Chile.

2 Equipo de Cirugía Plástica, Servicio de Cirugía, Hospital de Niños Roberto del Río.

Santiago, Chile.

\begin{abstract}
\section{Evaluation of donor site in auricular reconstruction of microtia with supraperichondrial costal cartilage harvest technique}

Aim: To assess the presence of donor site sequel of patients undergoing ear reconstruction with costal cartilage harvest, using supraperichondrial technique. Methods: Cross-sectional study; patients under 15 years. The cartilages were harvested with perichondrium. Three observers performed donor site assessment; they were independent to this study (validated Strasser score: deformity, asymmetry, contour and scar). Children and their mothers were applied satisfaction survey. Analysis of the association between variables: nonparametric tests. Results: 19 reconstructions, 18 patients, age 8 (6-15) years, follow-up 50 (14-96) months; number of rib cartilage harvested 3 (2-4) units. Expert assessment: excellent in 2 (10.5\%) cases, good in 10 $(52.6 \%)$, regular in $7(36.8 \%)$. Most mothers and patients rated the result as good. There was no significant difference in the score, with respect to the number of harvested costal cartilages. To separate them by age, poorer results were obtained at surgery before 10 years of age than in older $(p<0.03)$. Conclusion: Ear reconstruction and supraperichondrial technique for costal cartilage allows obtain good results in donor site. There is high satisfaction when evaluated by the patient and his mother, as well as observers. The result is not dependent on the number of harvested cartilage, but is related to age at surgery, best results were obtained in patients operated over 10 years of age.

Key words: Microtia, auricular reconstruction, costal cartilage.

\section{Resumen}

Objetivo: Evaluar presencia de secuela en zona dadora de pacientes sometidos a reconstrucción auricular con cartílago costal con técnica suprapericóndrica. Material y Método: Corte transversal; pacientes menores de 15 años. Cartílagos fueron levantados con pericondrio. Evaluación de zona dadora fue realizada

\footnotetext{
*Recibido el 22 de diciembre de 2014 y aceptado para publicación el 23 de agosto de 2015 .

Conflictos de interés: Ninguno

Correspondencia: Dr. Ricardo Yáñez ryanezm@med.puc.cl
} 
por tres observadores independientes (escala validada de Strasser: deformidad, asimetría, contorno y cicatriz). A niños y madres se les aplicó encuesta de satisfacción. En el análisis de la asociación entre variables se utilizan pruebas no paramétricas. Resultados: 19 reconstrucciones, 18 pacientes, mediana edad 8 (6-15) años, seguimiento 50 (14-96) meses, número de cartílagos costales obtenidos 3 (2-4) unidades. Evaluación de expertos: excelente en $2(10,5 \%)$ casos, bueno en 10 (52,6\%) y regular en $7(36,8 \%)$. La mayoría de las madres y pacientes calificó como bueno el resultado. No hubo diferencia significativa en el puntaje con respecto al número de cartílagos costales cosechados. Al separarlos por edad se obtuvo peores resultados en los intervenidos antes de los 10 años que en mayores $(\mathrm{p}<0,03)$. Conclusión: La reconstrucción auricular con cartílago costal y técnica suprapericóndrica, permite obtener buenos resultados en zona dadora. Existe alta satisfacción al ser evaluada por el paciente y su madre, así como por los observadores. El resultado no es dependiente del número de cartílagos; sí en relación a la edad de la cirugía: mejores resultados en pacientes operados sobre los 10 años de edad, lo que concuerda con otros estudios.

Palabras clave: Microtia, reconstrucción auricular, cartílago costal.

\section{Introducción}

La reconstrucción auricular en microtia es de elevada complejidad quirúrgica. En los últimos años, los avances en la técnica han permitido lograr resultados de mayor calidad ${ }^{1-4}$. El cartílago costal sigue siendo, sin discusión, el material de elección para la construcción de la nueva estructura o "molde", uno de sus principales inconvenientes es la deformidad de contorno que puede producir en la zona dadora en pacientes en crecimiento. Se han descrito distintas técnicas de obtención del cartílago con el fin de disminuir dicha morbilidad ${ }^{5,6}$. La técnica suprapericóndrica es aquella en que la cosecha del cartílago incluye el pericondrio y la subpericóndrica la que lo preserva, lo cual implica una dificultad quirúrgica mayor y su objetivo sería mantener en el paciente tejido con capacidad regenerativa. Existe poca literatura médica orientada a estudiar este aspecto y los resultados han sido controversiales con respecto al resultado estético y a la disminución de las secuelas?.

Nuestro objetivo es evaluar estéticamente a largo plazo la zona dadora de pacientes sometidos a técnica suprapericóndrica, analizando su relación con el número de cartílagos costales obtenidos y la edad del paciente al momento de la cirugía.

\section{Material y Método}

Estudio de corte transversal. Fueron analizados todos los pacientes menores de 15 años de edad que asistían a control ambulatorio de reconstrucción auricular por microtia durante un período observacional de 3 meses y que tuvieran, al menos, 12 meses de postoperado.

Cada cartílago fue obtenido de manera suprapericóndrica, obteniendo un número variable de cartílagos costales del sexto a noveno, según la preferencia del cirujano. Músculo, fascia y tejido subcutáneo fueron cerrados por planos con material de sutura reabsorbible.

A los pacientes se les tomó fotografías estándar de la región torácica en visión anterior, lateral y oblicua (previa autorización por medio de consentimiento informado) para compararlas con las preoperatorias. Estos registros fueron evaluados por tres observadores calificados e independientes al estudio, que no tenían información alguna respecto a detalles de la cirugía realizada, usando escala validada de Strasser que mide malposición, deformidad, asimetría, contorno y calidad de la cicatriz ${ }^{8}$. Se objetiva por medio de puntaje en el cual 0 puntos corresponde a excelente, 1 a 4 puntos es bueno, 5 a 14 puntos es regular y 15 o más es malo. A los niños y a sus madres se les aplica encuesta de satisfacción sobre el resultado estético de la zona dadora debiendo categorizarlo como malo-regular-bueno-perfecto. Para evitar influencias, se realizó por separado y en privado a madre e hijo/a.

Para variables categóricas se empleó frecuencia y porcentaje, en tanto que para variables continuas mediana y rango o promedio y desviación estándar según correspondiese. La asociación entre variables fue analizada con coeficiente de correlación de Spearman. Para muestras no paramétricas se utilizó prueba de Kruskal-Wallis.

\section{Resultados}

La serie incluyó 18 pacientes con un total de 19 reconstrucciones auriculares siendo mayormente hombres $\mathrm{n}=14(78 \%)$. La edad al momento de la cirugía fue de 8 años (rango: 6-15 años). El tiempo transcurrido desde la cirugía a nuestra evaluación fue de 50 meses (rango: 14-96 meses). La mediana de costillas levantadas fue de 3 unidades (rango, 2-4 costillas) (Tabla 1). 
El resultado de la evaluación de los observadores según la encuesta de Strasser fue excelente en 2 $(10,5 \%)$ casos, bueno en $10(52,6 \%)$ y regular en $7(36,8 \%)$ (Figura 1). El resultado de la encuesta de satisfacción a las madres y a los pacientes se

Tabla 1. Características de los pacientes evaluados

\begin{tabular}{|ll|}
\hline Características & $\mathbf{n}=\mathbf{1 8}$ pacientes \\
\hline Edad (mediana, rango) & $8(6-15)$ años \\
\hline Género (mujeres : hombres) & $14: 4$ \\
\hline$N^{o}$ costillas 2 & $3(16,6 \%)$ \\
3 & $10(55,5 \%)$ \\
4 & $5(27,8 \%)$ \\
Seguimiento (mediana, rango) & $50(14-96)$ meses \\
\hline
\end{tabular}

distribuyó como muestra la Tabla 2, donde la mayoría catalogó el resultado como bueno (65 y $60 \%$ respectivamente).

Al realizar el análisis del resultado de la encuesta de Strasser según número de cartílagos costales resecados, pudimos apreciar que no existe diferencia significativa entre puntajes obtenidos en los distintos grupos $(\mathrm{p}=0,45)$ (Figura 2), también se observó que la correlación entre número de cartílagos y puntaje obtenido no fue significativa con un $\mathrm{r}^{2}=0,044 \mathrm{y}$ $\mathrm{p}=0,42$ (Figura 3).

Dado que las últimas publicaciones mencionan un peor resultado en niños intervenidos antes de los 10 años, separamos la muestra en dos grupos etarios y constatamos que los pacientes que fueron intervenidos antes de los 10 años tuvieron un puntaje de Strasser de 4,5 puntos (rango: 0-8 puntos) y los de 10 años o más lograron una mediana de 1 punto (rango, 0-3 puntos) siendo esta diferencia estadísticamente significativa $(\mathrm{p}<0,033)$ (Figura 4).

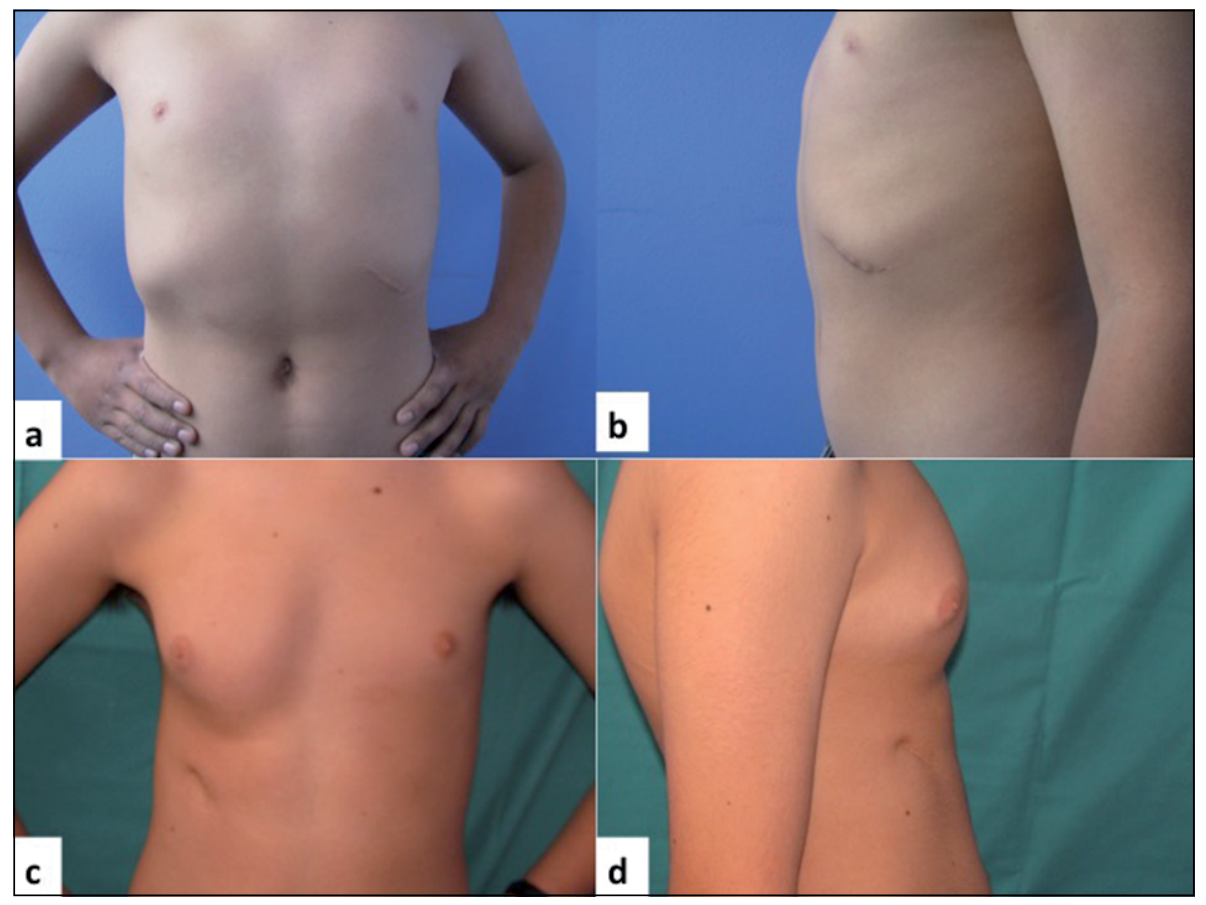

Figura 1. Aspecto de zona dadora a dos años de la cirugía; a y b corresponden a un paciente intervenido a los 12 años de edad, con un resultado adecuado; c y d corresponden a un paciente intervenido a $\operatorname{los} 8$ años de edad con un resultado insatisfactorio.

Tabla 2. Distribución de resultados de encuesta de satisfacción del resultado estético de la zona dadora, aplicada al paciente y a la madre

\begin{tabular}{|c|c|c|c|c|}
\hline & Excelente & Bueno & Regular & Malo \\
\hline Paciente & $10 \%$ & $60 \%$ & $25 \%$ & $5 \%$ \\
\hline Madre & $10 \%$ & $65 \%$ & $20 \%$ & $5 \%$ \\
\hline
\end{tabular}



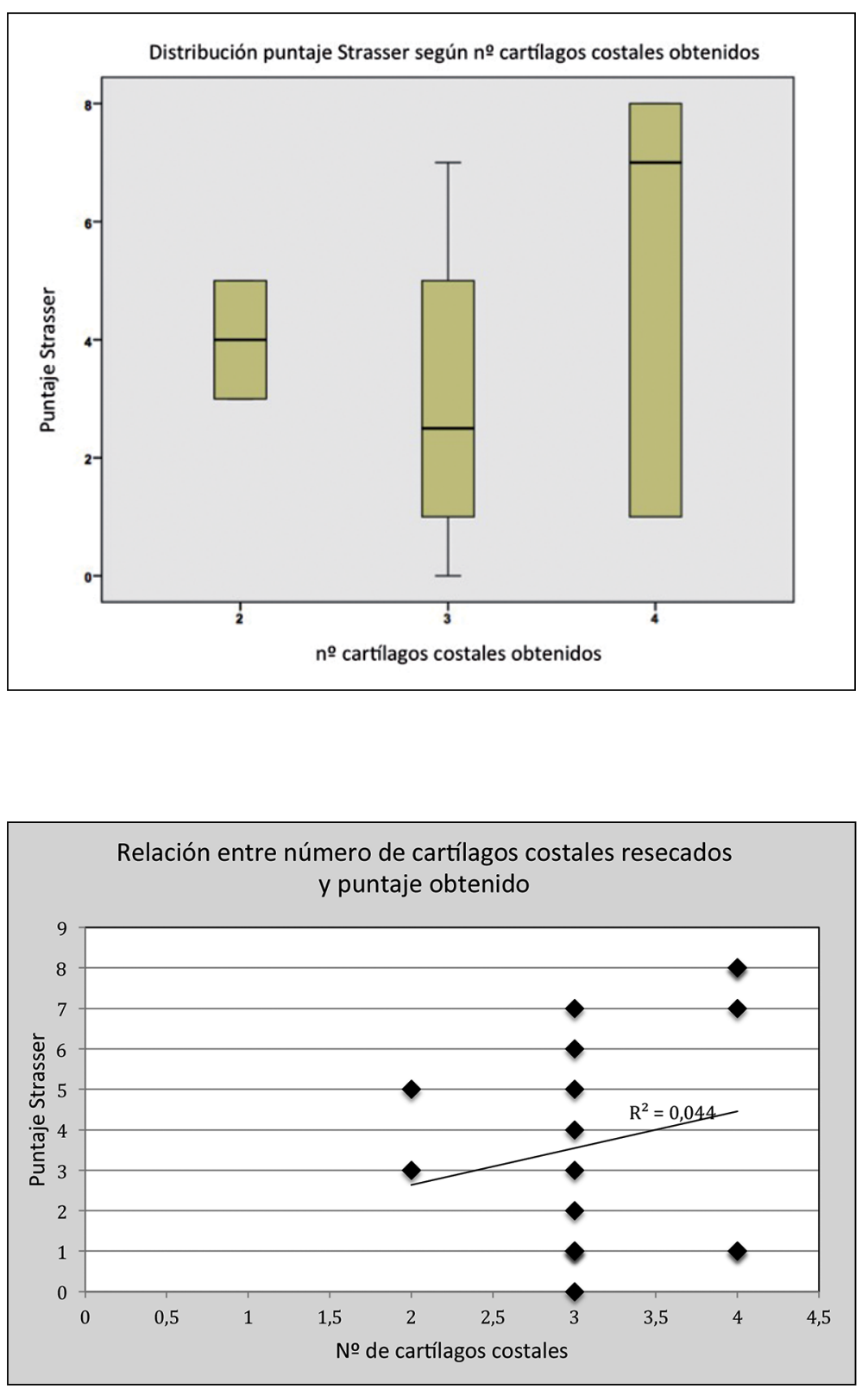

Figura 2. Distribución de puntajes obtenidos en encuesta de Strasser según número de costillas resecadas.
Figura 3. Relación entre costillas resecadas y puntaje obtenido en encuesta de Strasser. $\mathrm{r}^{2}=0,044$ y $\mathrm{p}=0,42$.

\section{Discusión}

La mayoría de las publicaciones sobre reconstrucción de microtia están orientadas a variaciones sobre la técnica quirúrgica destinadas a lograr un mejor resultado de la oreja reconstruida. Muy poco, sin embargo, podemos encontrar en relación a las complicaciones de mediano o largo plazo. Este estudio evaluó objetivamente la morbilidad tardía de la zona dadora de cartílago costal para reconstrucción auricular con técnica suprapericóndrica; considerando para ello la opinión del paciente, su madre y observadores calificados externos.

Los resultados arrojaron una alta satisfacción en relación a la apariencia de dicha zona al ser evaluada por el paciente o por su madre: $60 \%$ y el $65 \%$ respectivamente lo calificó como bueno. Asimismo, los observadores independientes en su mayoría lo calificaron como buena y una minoría como regular.

Durante años se ha asumido que la preserva- 


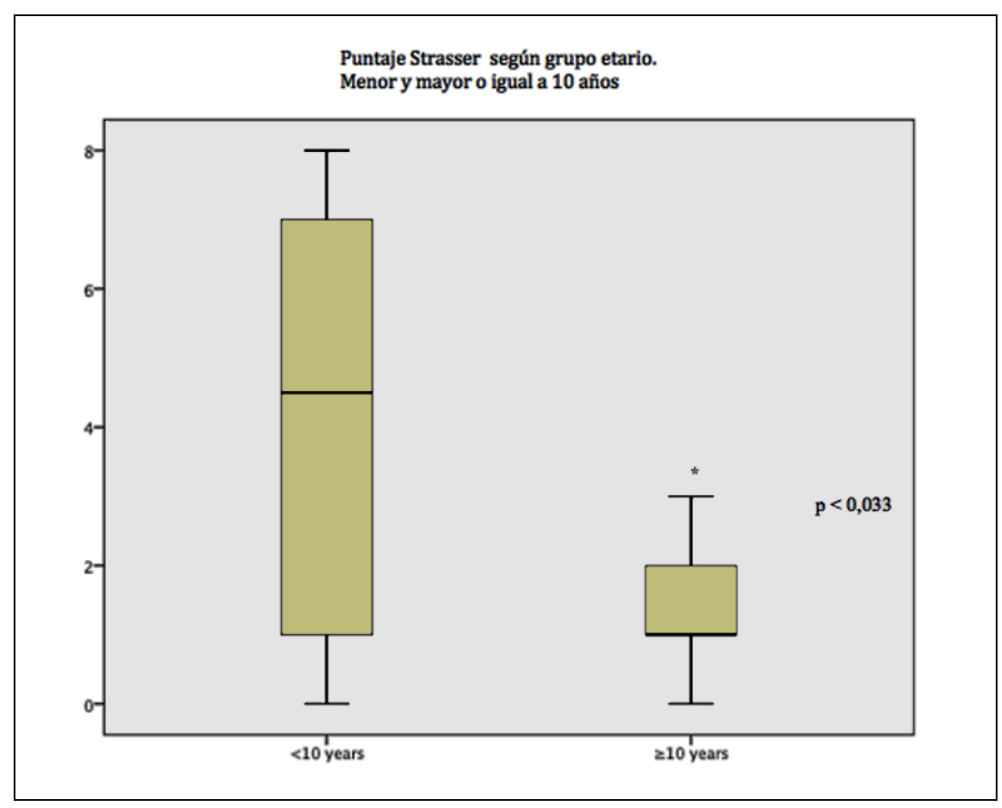

Figura 4. Distribución de puntajes obtenidos en encuesta de Strasser según grupo etario. ción del pericondrio ayudaría a la regeneración de cartílago costal, pero sin evidencia científica que sustente aquello. Nagata ${ }^{6}$, propone el año 2007 cerrar el pericondrio con pequeños bloques de $2 \mathrm{~mm}$ de los excedentes de cartílago tomado dentro de él, con lo cual "no tendría alteración de la parrilla costal", pero esto basado solamente en apreciación visual del propio cirujano. Gleen, reprodujo exactamente la técnica de dicho autor reportando que sí existen alteraciones medidas mediante TC $3 \mathrm{D}$, las cuales son mayores en los pacientes en crecimiento?.

No obtuvimos diferencia significativa al analizar el número de costillas cosechadas $(2,3$ o 4$)$ versus la presencia de secuela; sí obtuvimos diferencia cuando la cirugía se realizó en menores de 10 años. Esto último se correlaciona con lo publicado por Ohara ${ }^{10}$, quien encontró que la deformación es 3 veces más frecuente en este grupo etario. Nagata publica en la misma línea por lo que sólo en casos muy seleccionados realiza el procedimiento bajo los 10 años de edad. Importante es destacar que estos dos autores preservan el pericondrio a diferencia de nuestra serie, obteniendo todos los mismos resultados. Por otro lado, Glenn aporta a la discusión al comparar mediciones topográficas pre y postoperatorias en TC 3D y comprueba que en adultos sometidos a reconstrucción, las alteraciones anatómicas entre el lado operado y el lado sano, son menores a las del paciente en crecimiento, especialmente en sentido sagital. Thomson, en 88 evaluaciones de zonas da- doras, encontró deformación importante del tórax en $33 \%$ de aquellos niños operados entre 2-3 años versus $8 \%$ en quienes fueron sometidos a la misma cirugía entre 6-12 años ${ }^{11}$. Uppal, cosecha los cartílagos de la misma forma que nosotros, obteniendo aparentemente buenos resultados, aunque su evaluación del contorno no es tan objetiva como la que hace de la cicatriz residual ${ }^{12}$.

Los resultados obtenidos en pacientes con toma de cartílago con técnica suprapericóndrica fueron buenos, similares a los obtenidos con técnica subpericóndrica con preservación de cartílago de otras series, siendo esta última técnica de una mayor complejidad y requiere una mayor curva de aprendizaje para lograr buenos resultados.

Por lo anterior, nos parece más interesante la discusión que proponen varios autores en los últimos años de centrar la atención en la edad quirúrgica más que la preservación o no del pericondrio ${ }^{9-12}$.

Cabe señalar, de todas formas, que nuestro estudio es descriptivo, con un $n$ pequeño, y no tenemos grupo control o al que se le haya practicado otra técnica que nos permita realizar una comparación entre ellos, por lo que a partir de estos datos no se podría concluir al respecto; sin embargo, es interesante poder contar con este estudio de manera inicial para evaluar los resultados a largo plazo en un futuro análisis, donde sería interesante conocer el estado de la zona dadora una vez terminado el período de crecimiento de los niños, es decir, una vez que cumplan 18 años de edad. 


\section{Referencias}

1. Wilkes G, Wong J, Guilfoyle R. Microtia Reconstruction. Plast Reconstr Surg. 2014;134:464e-479e.

2. Firmin F. State-of-the-art autogenous ear reconstruction in cases of microtia. Adv Otorhinolaryngol. 2010;68:2552.

3. Firmin F, Marchac A. A novel algorithm for autologous ear reconstruction. Semin Plast Surg. 2011;25:257-64.

4. Brent B. Technical advances in ear reconstruction with autogenous rib cartilage grafts: Personal experience with 1200 cases. Plast Reconstr Surg. 1999;104:319-34.

5. Kawanabe Y, Nagata S. A New Method of Costal Cartilage Harvest for Total Auricular Reconstruction: Part I. Avoidance and Prevention of Intraoperative and Postoperative Complications and Problems. Plast Reconstr Surg. 2006;117:2011-8.

6. Kawanabe Y, Nagata S. A new method of costal cartilage harvest for total auricular reconstruction: part II. Evaluation and analysis of the regenerated costal cartilage. Plast Reconstr Surg. 2007;119:308-15.

7. Fattah A, Sebire N, Bulstrode N. Donor site reconstitu- tion for ear reconstruction. J Plast Reconstr Aesth Surg. 2010;63:1459-65.

8. Strasser EJ. An objective grading system for the evaluation of cosmetic surgical results. Plast Reconstr Surg. 1999;104:2282-5.

9. Glenn C, Wallace Ch, Mao HY, Wang CJ, Chen YA, Chen PK, et al. Three-dimensional computed tomography reveals different donor-site deformities in adult and growing microtia patients despite total subperichondrial costal cartilage harvest and donor-site reconstruction. Plast Reconstr Surg. 2014;133:640-51.

10. Ohara K, Nakamura K, Ohta E. Chest wall deformities and thoracic scoliosis after costal cartilage graft harvesting. Plast Reconstr Surg. 1997;99:1030-6.

11. Thomson HG, Kim TY, Ein SH. Residual problems in chest donor sites after microtia reconstruction: A longterm study. Plast Reconstr Surg. 1995;95:961-8.

12. Uppal RS, Sabbagh W, Chana J, Gault DT. DonorSite Morbidity after Autologous Costal Cartilage Harvest in Ear Reconstruction and Approaches to Reducing Donor-Site Contour Deformity. Plast Reconstr Surg. 2008;121:1949-55. 